\title{
Energies of ions in water and nanopores within Density Functional Theory
}

\author{
Kevin Leung ${ }^{1 *}$ and Martijn Marsman ${ }^{2}$ \\ ${ }^{1}$ Sandia National Laboratories, MS 1415, Albuquerque, NM 87185, kleung@sandia.gov \\ ${ }^{2}$ Institut fuer Materialphysik, Universitat Wien, Sensengasse 8/12, A-1090 Wien, Austria
}

(Dated: November 1, 2018)

\begin{abstract}
Accurate calculations of electrostatic potentials and treatment of substrate polarizability are critical for predicting the permeation of ions inside water-filled nanopores. The ab initio molecular dynamics method (AIMD), based on Density Functional Theory (DFT), accounts for the polarizability of materials, water, and solutes, and it should be the method of choice for predicting accurate electrostatic energies of ions. In practice, DFT coupled with the use of periodic boundary conditions in a charged system leads to large energy shifts. Results obtained using different DFT packages may vary because of the way pseudopotentials and long-range electrostatics are implemented. Using maximally localized Wannier functions, we apply robust corrections that yield relatively unambiguous ion energies in select molecular and aqueous systems and inside carbon nanotubes. Large binding energies are predicted for ions in metallic carbon nanotube arrays, while with consistent definitions $\mathrm{Na}^{+}$and $\mathrm{Cl}^{-}$energies are found to exhibit asymmetries comparable with those computed using non-polarizable water force fields.
\end{abstract}

\section{INTRODUCTION}

As computational power increases, Density Functional Theory (DFT)-based aqueous phase ab initio molecular dynamics (AIMD) simulations are becoming more widely used. DFT accounts for the polarizability of water molecules and can predict ion hydration structures significantly different from those computed using nonpolarizable force fields. It also accounts for the polarizability of synthetic nanopores like carbon nanotubes 1.2 and the surface charge/dipole distribution-induced electrostatic potential inside inorganic nanopores, $\frac{3.4}{.4}$ which may strongly affect ion permeation, rejection, partitioning, and exchange in these water-filled systems. The ability to use AIMD to compare the intrinsic free energies of ions in bulk water and inside water-filled nanopores may revolutionize our understanding and modeling ${ }^{5,6}$ of ion transport in nanopores. The predictions can potentially be tested using recently synthesized membranes made of vertically aligned carbon nanotube $e^{7,8}$ or functionalized silica nanopores $\stackrel{9}{\underline{9}}$ But up to now, when charge states of simulation cells are varied in the liquid phase, as when adding an ion to a simulation cell, the anomalous average, absolute, "intrinsic" electrostatic potential $\phi_{\sigma} \underline{10,11,12,13}$ has hindered direct AIMD determination of energies $\underline{14,15,16}$

In solid state DFT calculations, when Fourier transform or Ewald techniques are used to compute long range electrostatic interactions in periodically replicated unit cells, it has been widely accepted that $\phi_{o}$ is in general ambiguous: ${ }^{10,11,12,13}$ So is the energy incurred $q \phi_{o}$ when adding an ion of charge $q$ to a charge neutral simulation cell. Thus, successful estimates of the energy difference between charged species in vacuum and those embedded in a solid, such as the work function of electrons 17 or in electrochemistrical applications $\stackrel{18}{\underline{18}}$ have generally involved computing the difference in electrostatic poten- tials across an explicit interface in a single simulation cell. Since this difference depends on the interface, which is not always easily specified in complex systems, methods to reference the intrinsic electrostatic potential in solids to some known values, like the vacuum or Fermi level, have generated considerable interest. 13,19,20,21

In contrast, in physical chemistry, techniques for using classical force fields to compute the intrinsic free energy of ions in liquid water have been well established and widely applied, $22,23,24,25,26,27,28,29,30,31,32$ Here the electrostatic component of the ion hydration free energy is computed using thermodynamic integration (TI) or free energy perturbation (FEP) techniques ${ }^{33}$ It is added to the short-ranged repulsion and van der Waal's contributions, which are often represented by Lennard-Jones terms.

Formally, the predicted ion hydration free energies can be compared with thermodynamic estimates of the absolute hydration free energies of ions which are based on solvation and/or gas phase ion cluster data ${ }^{34,35}$ To this end, the pure solvent contribution to the water-vapor electrostatic "surface potential" $36,37,38,39 \Delta \phi$ also needs to be computed. Separating the calculation into intrinsic and interfacial contributions is advantageous because electrostatic effects are additive, and the pure solvent $\Delta \phi$ depends only on the water force field used $\stackrel{38,39}{ }$ Many widely used water models yield $\Delta \phi$ on the order of $\sim 10 \mathrm{kcal} / \mathrm{mol}$ per proton charge $\frac{38,40,41}{}$ At water-material interfaces, $\Delta \phi$ will include the substrate contribution. At vacuummaterial interfaces, the substrate $\Delta \phi$ directly contributes to the work function and can be tested against photoelectric measurements $\frac{17.42}{1}$ As will be shown, incorporating parts of the substrate or pure solvent $\Delta \phi$ is crucial for a consistent definition of intrinsic DFT ion energies. This is our main interest in the $\Delta \phi$ term.

Note that there is also an ionic contribution to aqueous phase $\Delta \phi$ which does not vanish even at infinite 


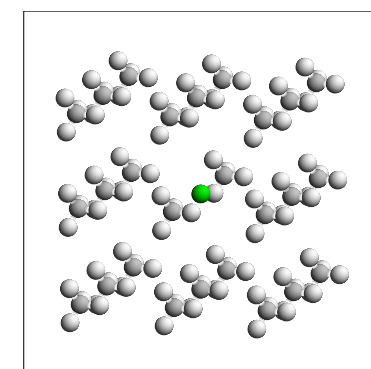

(a)

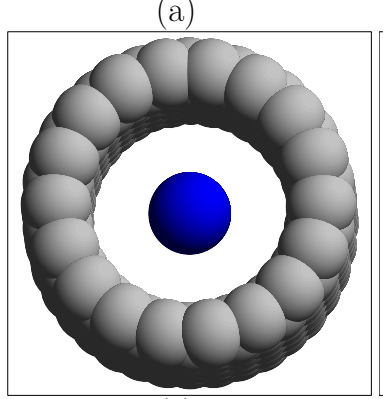

(c)

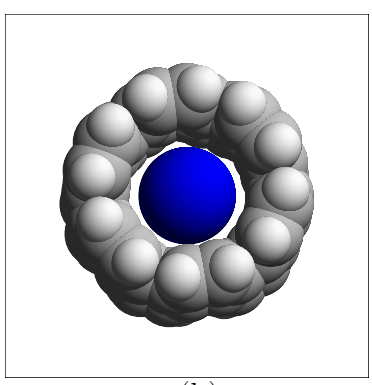

(b)

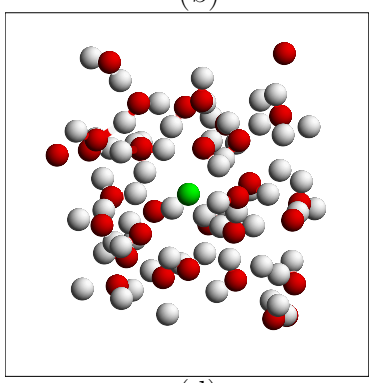

(d)
FIG. 1:

Samples of the model systems examined in this work. (a) $\mathrm{CH}_{4}$ molecular crystal; (b) (6,6) SWNT fragment; (c) infinitely long $(18,18)$ SWNT; (d) liquid water. The $\mathrm{Na}^{+}$ $\left(\mathrm{Cl}^{-}\right)$ion is depicted in blue (green).

ion dilution $\stackrel{43}{ }^{2}$ Further, it has been suggested $\Delta \phi$ at the water-vapor interface cannot be measured ${ }^{36,44}$ Nevertheless, the combination of intrinsic ion free energy differences in bulk and confined water and the interfacial $\Delta \phi$ may force ions into or reject them from narrow nanopore entrances and lead to novel ion blockage behavior at finite electrolyte concentration. These effects will likely affect ionic currents through nanoporous membranes.

In this work, we will use DFT to compute the intrinsic energy of ions in some condensed phase systems without explicit reference to an interface, just as has been done for ions in water using classical force fields. This requires regularizing the DFT electrostatic potential, which will be performed in two different environments: dipolefree molecular solids and metallic carbon nanotubes, and bulk liquid water. See Fig. 1. The philosophies differ, but technically they both involve going beyond the wellknown charge-image and charge-neutralizing background correction $19,20,23$ and considering the "spherical second moment" electrostatic potential contributions of the condensed phase material. 13,19,37 Unlike pure Ewald representation of electrostatics, our corrections involve making physical choices, such as preserving the integrity of molecules and nanotubes. To facilitate this correction in liquid water, the electronic charge density is decomposed on to molecular centers using maximally localized Wannier functions $\underline{45,46}$ To compare with DFT ion hydration results, we will focus exclusively on classical force field simulations that also apply Ewald sums. However, issues arising from truncating the coulomb interaction $24,47,48$ may also be relevant to DFT calculations that do not use Ewald sums.

This paper is organized as follows. Section 2 briefly describes the computational methods. It reviews how $\phi_{o}$ may be referenced to the vacuum or a known interface value, and emphasizes and reconciles the approaches taken in the solid state physics and physical chemistry communities. The results are presented in Sec. 3, and Sec. 4 concludes the paper with further discussions. An appendix describes the construction of maximally localized Wannier functions within the projected augmented wave (PAW) framework $\underline{49,50}$

\section{METHOD AND THEORY}

\section{A. Systems without dipole moments}

We consider

$$
E_{\text {ion }}=E_{\text {ion+substrate }}-E_{\text {bare ion }}-E_{\text {substrate }} .
$$

In periodic boundary condition DFT calculations, $E_{\text {ion }}$ contains the generally unknown term $q \phi_{o}$, where $q$ is the charge of the ion. This ambiguity arises from the inherently long-ranged nature of the Coulomb interaction $V(\mathbf{r})=1 /|\mathbf{r}|$, whose Fourier representation $\hat{V}(\mathbf{k})=$ $4 \pi /|\mathbf{k}|^{2}$ is undefined at $\mathbf{k}=0$. Ewald-sum calculations almost universally and arbitarily set $\hat{V}(\mathbf{k}=\mathbf{0}) \propto \phi_{o}$ to zero.

In this work, we assume that any ion-containing periodically replicated simulation cell has a neutralizing background, in effect removing the monopole. At zero temperature, the correction to $E_{\text {ion }}$ due to the background and the image charge interactions has been well documented in the DFT literature $\underline{\underline{19}}$ It is

$$
E_{\text {ewald }}=q^{2} \alpha /(2 L \epsilon)
$$

in atomic units, where $\alpha$ is the Madelung constant, $L$ is the length of the cubic unit cell, and $\epsilon=\epsilon_{\infty}$ or $\epsilon_{o}$ depending on the application. When computing ion hydration free energy in liquids using classical force fields, a similar correction yield system size independent hydration free energies, $23,24,25,26,27,28,29,30,31$ Equation 2 does not remove the electric field arising from the periodic images, which is an area of active research 20,51 However, for the purpose of this work, we will assume the monopole issue is a solved problem, and will apply Eq. 2 and its generalization to non-cubic cells with the appropriate $\epsilon$. Instead, we focus on the correction to the intrinsic electrostatic potential which persists, and can be on the order of electron volts, even in the limit of a very large unit cell where $E_{\text {ewald }}$ becomes vanishingly small. The origin of this shift in $\phi_{o}$ is discussed below and is further illustrated in Sec. IIIA 
In general, a simulation cell exhibits finite dipole (d), quadrupole (the second rank tensor $\tilde{\mathbf{Q}}$ ), and "spherical second" (SSM, $\bar{Q}$ ) moments:

$$
\begin{aligned}
\mathbf{d} & =\int d \mathbf{r}\left(\mathbf{r}-\mathbf{R}_{c}\right) \rho(\mathbf{r}) \\
\tilde{\mathbf{Q}} & =(1 / 2) \int d \mathbf{r}\left(\mathbf{r}-\mathbf{R}_{c}\right):\left(\mathbf{r}-\mathbf{R}_{c}\right) \rho(\mathbf{r}) \\
\bar{Q} & =(1 / 3) \operatorname{Tr} \tilde{\mathbf{Q}}
\end{aligned}
$$

Here $\mathbf{R}_{c}$ is an arbitarily chosen center of the unit cell, $\rho(\mathbf{r})$ is the total charge density, and the integration over $\mathbf{r}$ in the unit cell includes both electronic and nuclear degrees of freedom,

$$
\int d \mathbf{r} \rho(\mathbf{r}) \rightarrow \int d \mathbf{r}_{\mathrm{e}} \rho_{e}\left(\mathbf{r}_{\mathrm{e}}\right)+\sum_{m \beta} q_{m \beta} \delta\left(\mathbf{r}-\mathbf{R}_{m \beta}\right),
$$

where $\beta$ denotes the nuclear site on molecule $m$, and $e$ stands for electrons.

Unit cells with non-zero $\mathbf{d}$ and or $\tilde{\mathbf{Q}}$ elements exhibit $\phi_{o}$ that depend on the surface terminations. Consider a macroscopic but finite object built from a periodically replicated unit cell, terminated in interfaces with the vacuum.$\underline{13}$ If the lowest multiple moment of the the unit cell is a monopole, dipole, or quadrupole, the center of the object ("crystal") experiences an electrostatic potential of the form $\int^{R} d r r^{2} f(r)$ exerted by the rest of the object. Here $|f(r)| \sim 1 / r, 1 / r^{2}$, or $1 / r^{3}$ respectively, and the vacuum region outside the object, $r>R$, is assigned an electrostatic potential of zero. The integral is not uniformly convergent at large $R$, and thus $\phi_{o}$ in unit cells at the center of the object depends on the shape of the object in addition to the nature of the interface ${ }^{13}$ In particular, if $|\mathbf{d}| \neq 0, \tilde{\mathbf{Q}}$ will depend on the choice of $\mathbf{R}_{c}$, and there will be an $\mathbf{d}$-dependent electric field across each unit cell, 10,11,12,13

Ewald methods, when applied with the "tin-foil" boundary conditions (almost universally the case in DFT calculations), effectively impose an infinite $\epsilon_{\infty}$ dielectric continuum boundary that eliminates $\mathbf{d}$ - and $\tilde{\mathbf{Q}}$-induced electric fields and potentials. As mentioned above, it sets $\phi_{o}=\hat{V}(\mathbf{k}=0)=0$ independent of $\mathbf{R}_{c} \cdot \underline{13}$ To report $E_{\text {ion }}$ in the absence of an interface, the "intrinsic" nonzero $q \phi_{o}$, which depends on physical choices of system boundaries, needs to be added to the Ewald-computed ion energy. Such a regularization of DFT electrostatic potential within Ewald sum calculations has been carried out in Ref. 19 for an isolated ion in the gas phase. The importance of this correction has not been sufficiently emphasized in solid or liquid systems.

We first consider special cases where $\mathbf{d}$ vanishes or can be chosen to vanish, and obvious physical choices of simulation cell boundaries can be defined. Such systems include ions inside a crystal made up of molecules without permanent dipole moments, and ions embedded in carbon nanotubes. Consider the spherical second moment (SSM) contribution in these systems. This term does not appear in real space multipole expansions of the electrostatic potential exerted by an arbitary charge distribution ${ }^{53}$ But when Ewald sums arbitarily set $\phi_{o}=0$, an SSM-dependent constant shift arises. This shift is undone by adding: $\underline{13,19}$

$$
\phi_{\mathrm{SSM}}\left(\mathbf{R}_{c}\right)=-(2 \pi / 3 \Omega) \int d \mathbf{r} \rho(\mathbf{r})\left(\mathbf{r}-\mathbf{R}_{c}\right)^{2},
$$

where $\Omega$ is the unit cell volume. If $\rho(\mathbf{r})$ can be decomposed into molecular contributions labeled by "m" and centered on molecular centers $\mathbf{R}_{m}$, such that $\rho(\mathbf{r})=$ $\sum_{m} \rho_{m}(\mathbf{r})$, then

$$
\begin{aligned}
\phi_{\mathrm{SSM}}\left(\mathbf{R}_{\mathrm{c}}\right) & =-(2 \pi / 3 \Omega) \sum_{m}\left[\int d \mathbf{r} \rho_{m}(\mathbf{r})\left(\mathbf{r}-\mathbf{R}_{m}\right)^{2}\right. \\
& \left.-2 \int d \mathbf{r}\left(\mathbf{R}_{c}-\mathbf{R}_{m}\right) \cdot\left(\mathbf{r}-\mathbf{R}_{m}\right) \rho_{m}(\mathbf{r})\right],
\end{aligned}
$$

where we have used the fact that each molecule has no net charge. The position of the ion, if present, is assumed to coincide with $\mathbf{R}_{c}$, which permits the use of Eq. [7 without adding monopole terms. If each molecule also possesses a zero dipole moment, the second term vanishes, and Eq.7 simplifies to

$$
\phi_{\mathrm{SSM}}=-(2 \pi / 3 \Omega) \sum_{m} \int d \mathbf{r} \rho_{m}(\mathbf{r})\left(\mathbf{r}-\mathbf{R}_{m}\right)^{2} .
$$

In this case, $\phi_{\mathrm{SSM}}$ no longer depends on $\mathbf{R}_{c}$. A system of identical, non-interacting molecules without dipole $\left(\mathbf{d}_{m}=0\right)$ or quadrupole moments, $\left(\left[\tilde{\mathbf{Q}}_{\mathbf{m}}-1 / 3 \operatorname{Tr} \tilde{\mathbf{Q}}_{\mathbf{m}}\right]_{i j}=\right.$ $0, i \neq j), \tilde{\mathbf{Q}}_{m}=(1 / 2) \int d \mathbf{r} \rho_{m}(\mathbf{r})\left(\mathbf{r}-\mathbf{R}_{m}\right):\left(\mathbf{r}-\mathbf{R}_{m}\right)$, would then yield a $\phi_{\mathrm{SSM}}$ proportional to the particle density and the molecular-centered term $\int d \mathbf{r} \rho_{m}(\mathbf{r})\left|\mathbf{r}-\mathbf{R}_{m}\right|^{2}$. The absence of $\mathbf{d}_{m}$ means that $\tilde{\mathbf{Q}}_{m}$ is also independent of $\mathbf{R}_{m}$.

Several points are worthy of note. (1) Unlike pure Ewald summation, applying SSM corrections amounts to making physical choices of system boundaries. The electron density $\rho_{e}(r)$ is not uniquely assigned to a simulation cell, but can be varied via "spreading transformations." $\underline{54}$ For example, one can split the $\rho_{e}(r)$ of a molecule which straddles the unit cell boundary, creating two or more charge fragments; the $\phi_{\mathrm{SSM}}$ correction, now no longer decomposable into intact molecular contributions, will depend on how this splitting is performed. Physically meaningful interfaces consist of intact molecules without splitting of molecular orbitals across unit cell boundaries. When modeling ions in nanotubes, the choice of boundaries will clearly affect the $q \phi_{\mathrm{SSM}}$ corrections to ion binding energies inside the pores. Here the simulation cells should preserve the $\rho_{e}(r)$ of intact SWNT in the lateral 
directions. Splitting of the continuous $\rho_{e}(r)$ along the axial direction is necessary, but intuitive choices can still be made to demarcate $\rho_{e}(r)$ within each unit cell of an infinitely long SWNT, guided by symmetry considerations. In covalently bonded solids like silicon, the choice of the unit cell is less obvious. (2) Note that carbon nanotubes are generally chemically modified at their two open ends, leading to surface contributions that may need to be added to the computed intrinisic electrostatic potential when predicting ion transport through synthetic nanoporous membranes, $\frac{7,8,9}{1}$ Ionic contributions will also be present $\underline{\underline{43}}$ (3) $\phi_{\mathrm{SSM}}$ depends on whether core electrons or pseudopotentials are used via Eq. 6. Correcting $\phi_{o}$ with $\phi_{\mathrm{SSM}}$ is thus critical for comparing intrinsic ion energies computed using different DFT codes and methods. (4) For unit cells without dipole moments, but with finite quadrupole moments, $[\tilde{\mathbf{Q}}-1 / 3 \operatorname{Tr} \tilde{\mathbf{Q}}]_{i i} \neq 0, \phi_{o}$ still mathematically depends on the shape of the macroscopic system, but not on unphysical considerations such as whether core electrons are used. (5) For isolated ionic species in vacuum, $q \phi_{\mathrm{SSM}}$ vanishes when $\Omega \rightarrow \infty$. In condensed phase systems, where finite electronic and nuclear charge densities persist in all space, $q \phi_{\mathrm{SSM}}$ becomes $\Omega$-independent for large simulation cells, and is roughly proportional to the atomic density. As will be seen, it can result in a several volt correction to the DFT-computed $\phi_{o}$. (6) As will be discussed, classical force fields also exhibit $q \phi_{\mathrm{SSM}}$ corrections to the electrostatic potential, albeit smaller ones that contain no ambiguity associated with "core electrons" contributions.

\section{B. Ions in liquid water}

We also consider the energy of ions in bulk liquid water, where Eq. 1 is averaged over the AIMD trajectory. Here $E_{\text {ion }}$ is not the ion hydration enthalpy. It does not account for the solvent reorganization penalty arising from molecular motion, and is instead related to the value of the integrand at the end point of the thermodynamic integration formula where the ion charge $q_{\lambda}$ is gradually increased from zero to $q 2^{23}$ See Sec. IIIC, See Sec. III C. However, regularizing $\phi_{o}$ is still crucial for correcting $E_{\text {ion }}\left(q_{\lambda}\right)$ along the entire $q_{\lambda}$-integration path. As such, our work lays the foundation for future AIMD thermodynamic integration calculations.

In general, a simulation cell containing an instantaneous configuration of liquid water has non-vanishing dipole and quadrupole moments. Each water molecule also has finite $\mathbf{d}_{m}$ and $\tilde{\mathbf{Q}}_{m}$ elements. The finite $\mathbf{d}_{m}$ means that $\tilde{\mathbf{Q}}_{m}$ now depends on the choice of the molecular center ${ }^{53}$ Despite this, following formulations developed for classical water force fields (see below), $q \phi_{\mathrm{SSM}}$ (Eq. 8) is still applicable because neither the vectors $\left(\mathbf{R}_{c}-\mathbf{R}_{m}\right)$ nor the molecular axes (implicit in $\int d \mathbf{r}$ ) has preferred orientations, and the second term in Eq. 7 averages to zero in liquid environments. We will collapse the electron density into molecular contributions via the maximally localized Wannier function approach, $\underline{45,46}$ taking care to preserve the integrity of $\mathrm{H}_{2} \mathrm{O}$ molecules and not cleaving orbitals across unit cell boundaries.

Formally, comparing predicted absolute ion free energies with thermodynamic estimates based on solvation and/or gas phase ion cluster data ${ }^{34,35}$ requires adding the pure solvent contribution to $\Delta \phi$ at the water-vapor interface (henceforth simply referred to as $\Delta \phi$ in this section). The $\Delta \phi$ formulation is well established in the classical force field literature: $\underline{36.37}$

$$
\begin{aligned}
\Delta \phi & =\left\langle 4 \pi\left[-(1 / 3) \Delta \rho \operatorname{Tr} \tilde{\mathbf{Q}}_{m}+\int_{z_{1}}^{z_{2}} \rho_{d}(z)\right]\right\rangle \\
& =\Delta \phi_{Q}+\Delta \phi_{d} \\
\rho_{d}(z) & =\left\langle\int d \mathbf{r} \rho_{m}(\mathbf{r}) \delta\left[z-\left(\mathbf{R}_{m}\right)_{z}\right]\left(\mathbf{d}_{m}\right)_{z}\right\rangle .
\end{aligned}
$$

Here $z$ is the direction perpendicular to the interface, $\Delta \rho$ is the difference between water and vapor densities, and $\rho_{d}(z)$ is the dipole density profile. $\mathbf{d}_{m}$ and $\tilde{\mathbf{Q}}_{m}$ are the dipole vector and quadrupole moment tensor for water molecule $m$. They are in fact independent of $m$ because of liquid phase self-averaging ("〈...〉"). Equation 9 would involve a sum over molecular species if more than one were present. Its derivation makes use of the lack of preferred molecular orientations, mentioned above. Although Refs. 36 and 37, which preceed Ref. 13, do not use the term "second spherical moment," the first term on the right side of Eq. 9 is clearly equivalent to $\phi_{\mathrm{SSM}}$ defined in Eq. 8.

Thus, the intrinsic ion hydration free energy computed using classical force fields is typically defined without the $q \phi_{\mathrm{SSM}}$ term, which is instead included as part of the pure solvent surface potential $\Delta \phi \underline{55,56,57}$ The numerical values of $\rho_{d}(z)$ and $\tilde{\mathbf{Q}}_{m}$ depend on the choice of the molecular center $\mathbf{R}_{m}$, but $E_{\text {ion }}+q \Delta \phi$ (or more precisely the free energy $\left.\Delta G_{\text {ion }}+q \Delta \phi\right)$ is independent of such choices $\underline{37}$

If we compute $q \Delta \phi$ using an AIMD simulation of the water-vapor interface $\underline{\underline{58}}$ and add it to an appropriately defined AIMD intrinsic ion hydration free energy, the result will be unambiguous. However, using AIMD to compute $\Delta \phi$ is not yet feasible because (a) large numerical noises are associated with the liquid-vapor interfacial fluctuations, and overly long and costly AIMD trajectories would be required to yield good statistics for $\Delta \phi$. (b) Widely used DFT functionals do not necessarily yield $1.0 \mathrm{~g} / \mathrm{cc}$ water density, $, 58,59$ rendering the use of Eq. 9 , which depends on the difference between bulk water and vapor densities $\Delta \rho$, problematic.

At present then, we believe AIMD predictions of ion free energies are most useful for comparison with and validation of classical force fields. This comparison requires that the $q \phi_{\mathrm{SSM}}$ correction be consistently included as part of the intrinsic ion energy, and not as part of $\Delta \phi$, 
for both AIMD and classical force field simulations. In fact, if they are both perfectly accurate and consistently defined (using the same molecular centers $\mathbf{R}_{m}$ ), DFT and classical force field calculations of $\Delta \phi_{d}$ (Eq. 10), and not just the intrinsic ion energies, should agree with each other. This is because $\rho_{d}(z)$ only depends on the spatial and orientational distributions of water molecules and their instantaneous dipole moments at the water-vapor interface. But $\Delta \phi$ will not be the focus of this work; our interest in this interfacial quantity is mainly to establish a consistent definition of $E_{\text {ion }}$ such that DFT, force field predictions, and thermodynamic data ${ }^{34.35}$ can be compared.

Recall that $E_{\text {ion }}$ neglects solvent reorganization cost arising from molecular motion - the dominant contribution to hydration in liquid water. Using Ewald-sums and non-polarizable classical force fields, this definition of $E_{\text {ion }}$ (or " $q \phi$ " in the notation of Ref. 23) becomes independent of the simulation cell size when it is corrected by twice the value of Eq. $2^{23}$ We will apply this correction to AIMD-predicted $E_{\text {ion }}$ to compare with force field results. Here the DFT electron density already "reorganizes" to accommodate the ion and introduces a slight ambiguity. The Ref. 23 correction will be shown to be reasonable by testing $E_{\text {ion }}$ convergence with system size. In contrast, the absolute ion hydration free energy $(\Delta G)$, a physical quantity, contains both electronic and nuclear solvent reorganization. It should be unambiguously cell-size independent when Eq. 2 is subtracted once $\frac{23}{2}$ As mentioned above, for ions inside carbon nanotubes, electronic relaxation dominates; we will freeze nuclear degrees of freedom and show that Eq. 2 suffices for convergence.

To recapitulate, we consider two types of systems: ions in dipole-free unit cells and in liquid water, both pertinent to ion penetration into water-filled nanopores. Different philosophies, traditionally associated with solid and liquid phases, are involved, but the $\phi_{\mathrm{SSM}}$ corrections (Eqs. [6 and 8) are crucial in both cases. For a system of dipole- and quadrupole-free molecules, the two viewpoints coincide. $\phi_{Q}$ (Eq. 9) becomes independent of the choice of molecular centers, $\rho_{d}(z)$ vanishes identically, and Eq. 9 reduces to Eq. 8. In this case, Eq. 9 or Eq. 8 directly references $E_{\text {ion }}$ to $\phi_{\text {vacuum }}=0$ through a material-vacuum interface that has no dipole contribution to $\Delta \phi$ (Fig. 1h).

\section{VASP calculations}

We apply the Vienna Atomistic Simulation Package (VASP) 50,60 and the standard VASP suite of ultrasoft (US) ${ }^{61}$ and projector-augmented wave (PAW) pseudopotentials (PP) $\stackrel{49,50}{=}$ US PP are used in AIMD simulations while static calculations utilize PAW PP. The PerdewWang (PW91) and Perdew-Burke-Ernzerhof (PBE) exchange correlation functionals ${ }^{62,63}$ are applied for ions in bulk liquid water and ions inside molecular solid/carbon nanotubes, respectively. These functionals generally yield similar predictions. The $\mathrm{Na}^{+} \mathrm{PP}$ does not include pseudovalent $p$ electrons. The AIMD time step is $0.25 \mathrm{fs}$, the cubic simulation cells have sizes of $9.9874 \AA$ $(12.509 \AA)$ for $32(64) \mathrm{H}_{2} \mathrm{O}$ molecules plus one ion, all proton masses are replaced by deuterium masses, and the plane-wave energy cutoff is set at $400 \mathrm{eV}$. The convergence criterion at each of the Born Oppenheimer time steps is $10^{-5} \mathrm{eV}$. The total energy is conserved to within $1 \mathrm{~K} / \mathrm{ps}(2 \mathrm{~K} / \mathrm{ps})$ for the $\mathrm{Cl}^{-}\left(\mathrm{Na}^{+}\right)$simulation cells, respectively. An elevated temperature of $\mathrm{T}=375 \mathrm{~K}$ is enforced using a Nose thermostat to alleviate water-overstructuring problems when applying the PW91 functional for AIMD simulations ${ }^{64,65,66}$ The trajectory lengths used, not counting pre-equilibration runs, are at least $20 \mathrm{ps}$. In one case $\left(\mathrm{Cl}^{-}\right.$in $64 \mathrm{H}_{2} \mathrm{O}$ box $)$, we actually use two different initial configurations obtained from classical force field molecular dynamics simulations, conduct two 10 ps AIMD trajectories, and combine the results. The mean $E_{\text {ion }}$ of the two AIMD segments are within $0.034 \mathrm{eV}$ of each other. In the aqueous phase calculations, $E_{\text {ion }}(\mathrm{Eq}$. 1) is sampled every 0.1 ps. $\Gamma$-point Brillouin zone sampling is applied in most cases, except that a $1 \times 1 \times 10 k$ Monkhorst-Pack grid is also used in convergence tests for infinitely long metallic carbon nanotubes.

The implementation of the maximally localized Wannier functions in VASP is limited to a $\Gamma$-point only sampling of the Brillouin zone. Details concerning the construction of maximally localized Wannier functions within VASP are provided in the Appendix.

To apply Eqs. 2 and 6 the VASP subroutine pot.F is slightly modified so that the electrostatic potential at $\mathbf{G}=0, \mathbf{k}=0$ reflects the total number of electrons (not the negative of the total pseudo-nuclear charge).

\section{RESULTS}

\section{A. Ions in molecular crystals}

We first consider two test cases of hypothetical simple cubic crystals made up of $\mathrm{Ar}$ atoms and $\mathrm{CH}_{4}$ molecules. A lattice constant of $5 \AA$ ensures that the molecules are well-separated. We construct a $3 \times 3 \times 3$ supercell, create a vacancy at the supercell center, and insert a $\mathrm{Na}^{+}$or $\mathrm{Cl}^{-}$ion there. The Ewald corrections (Eq. 2) to eliminate the effects of the neutralizing background cancel if we assume $\epsilon=1$ for both the isolated ion and the ion inside the molecular crystal vacancy 67 Centering the ion in this supercell ("unit cell") leads to zero net dipole and quadrupole moments. This physical choice of cell boundary allows unambiguous computation of $q \phi_{\mathrm{SSM}}$ using Eq. 6 (i.e., without decomposing $\rho(r)$ into molecular contributions). Furthermore, for a macroscopic crystal 


\begin{tabular}{||c|c|c|c||}
\hline system & cell size & $\mathrm{Na}^{+}$ & $\mathrm{Cl}^{-}$ \\
\hline $\mathrm{CH}_{4}(\mathrm{~s})$ & $15.0 \times 15.0 \times 15.0$ & $-0.22(-1.33)$ & $-0.33(+1.00)$ \\
$\mathrm{Ar}(\mathrm{s})$ & $15.0 \times 15.0 \times 15.0$ & $-0.12(-1.66)$ & $-0.20(+1.48)$ \\
$\mathrm{C}_{48} \mathrm{H}_{24}$ & $15.0 \times 15.0 \times 15.0$ & $-1.11(-2.48)$ & $-0.10(+1.51)$ \\
$\mathrm{C}_{48} \mathrm{H}_{24}$ & $20.0 \times 20.0 \times 20.0$ & $-1.18(-1.76)$ & $-0.15(+0.53)$ \\
$(6,6)$ & $14.8 \times 14.8 \times 14.8$ & $-2.85(-6.29)$ & $-1.77(+2.14)$ \\
$(6,6)$ & $14.8 \times 14.8 \times 22.2$ & $-2.86(-6.26)$ & $-1.80(+2.16)$ \\
$(18,18)$ & $17.0 \times 17.0 \times 17.0$ & $-2.20^{*}(-7.04)$ & $-1.09^{\dagger}(+4.11)$ \\
\hline
\end{tabular}

TABLE I: $E_{\text {ion }}$ inside molecular crystals and SWNT's, in units of eV. Simulation cell sizes are in $\AA^{3}$. The values in brackets are computed without the $q \phi_{\mathrm{SSM}}$ correction. ${ }^{*} \mathrm{~K}^{+}$rather than $\mathrm{Na}^{+}$is used in the $(18,18) \mathrm{SWNT}^{\dagger}$ overestimated due to a small amount of electron transfer (see text).

made up of this unit cell, any crystal-vacuum interface will not contribute to $\phi_{o}, \Delta \phi=0$, and our predicted $E_{\text {ion }}$ are unambiguously referenced to the vacuum value $\phi=0$.

No geometric relaxation of the lattice atoms is allowed after inserting $\mathrm{Na}^{+}$or $\mathrm{Cl}^{-}$. $E_{\text {bare ion }}$ for the isolated $\mathrm{Cl}^{-}$in a $(15 \AA)^{3}$ unit cell also exhibits a small $q \phi_{\mathrm{SSM}}=$ $-0.1 \mathrm{eV}$ using pseudopotential containing only $3 s$ and $3 p$ valence electrons. This is subtracted from the $\mathrm{Cl}^{-}$-plussubstrate $q \phi_{\mathrm{SSM}}$ term. With these corrections, we obtain $E_{\text {ion }}=-0.22(-0.33) \mathrm{eV}$ for $\mathrm{Na}^{+}\left(\mathrm{Cl}^{-}\right)$in the $\mathrm{CH}_{4}$ solid, and $E_{\text {ion }}=-0.12(-0.20) \mathrm{eV}$ in Ar solid. These values are reasonable. With these large band gap atoms/molecules, we expect small interactions between the ions and the molecular dipoles they induce in the substrate.

More significantly, adding $q \phi_{\mathrm{SSM}}$ leads to comparable $E_{\mathrm{ion}}$ for both ions. This is expected because the binding energy should be roughly proportional to $q^{2}$. In the absence of $q \phi_{\mathrm{SSM}}$ corrections, $\mathrm{Na}^{+}$and $\mathrm{Cl}^{-}$ions exhibit $E_{\text {ion }} \sim \pm 1 \mathrm{eV}$ in our model $\mathrm{CH}_{4}$ and Ar solids (see Table \). The large asymmetry between cations and anions, and the predicted repulsion of $\mathrm{Cl}^{-}$, mean that these uncorrected DFT energies are clearly unphysical. Table I implies that $\phi_{\mathrm{SSM}}>0$ in the DFT calculations. This is because nuclei are extremely localized in space whereas electrons are more diffuse. The true electrostatic potential $\phi_{o}$ averaged over the unit cell includes contributions inside atomic cores where $\phi>0$. Thus $\phi_{o}$ is positive definite inside the crystal, not zero as Ewald sums typically mandate. The position independent $\phi_{\mathrm{SSM}}$ term correctly accounts for this. Similar corrections have been applied to non-polarizable Ne solid and Ar fluid models using analytical atomic charge distributions $\underline{21,56}$

The $\phi_{\mathrm{SSM}}$ correction is a consideration whenever classical force fields with distributed charges and Ewald sums are used to compute intrinsic hydration free energies of ions ${ }^{68,69}$ Even without distributed charges, classical force field descriptions of molecules still exhibit such corrections. If the $\mathrm{C}$ and $\mathrm{H}$ atom sites in our $\mathrm{CH}_{4}$ solid carry fixed point charges of $-0.4 e$ and $+0.1 e$, $\phi_{\mathrm{SSM}}=-0.11$ volt. Adding $q \phi_{\mathrm{SSM}}$ will then yield the expected physical result, namely, that the ion energies in the vacancy site are almost zero when computed using non-polarizable force fields. Recall that $\phi_{\mathrm{SSM}}$ can alternatively be defined as part of the interface surface potential (Eq. 9) in force field calculations.

\section{B. Ions in carbon nanotube models}

Unlike the above molecular crystal test cases, the interactions of ions inside single wall carbon nanotubes (SWNT) are of significant technological interest. Narrow pore $(\sim 8 \AA$ diameter $)$ SWNT arrays have been proposed as osmotic membranes,,$\underline{5}$ and axially aligned SWNT's with larger pore diameters have recently been synthesized $\stackrel{7.8}{.}$ However, most molecular dynamics simulations of water-filled SWNT $3,5,6,52$ ignore SWNT

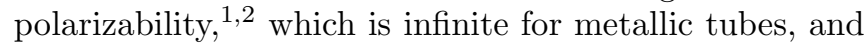
large polarizability-induced effects are expected.

Figure 10 depicts a proton-terminated $(6,6)$ SWNT fragment $\mathrm{C}_{48} \mathrm{H}_{24}$ situated in the center of a $15 \times 15 \times 15 \AA^{3}$ cubic unit cell. This finite-sized "molecule" has a finite band gap and no dipole moments. We place a $\mathrm{Na}^{+}$or a $\mathrm{Cl}^{-}$ion at the center of the $\mathrm{C}_{48} \mathrm{H}_{24}$ interior. Ewald corrections (Eq. 22) used to eliminate monopole contributions are again assumed to cancel for the bare ions and the ion-SWNT fragment complexes (see below). The net $E_{\text {ion }}$, computed without relaxing SWNT atoms, are predicted to be $-1.11 \mathrm{eV}$ and $-0.10 \mathrm{eV}$ for $\mathrm{Na}^{+}$and $\mathrm{Cl}^{-}$, respectively (Table I). Without $q \phi_{\mathrm{SSM}}$ (Eq. [6), they are erroneously predicted to be $-2.6 \mathrm{eV}$ and $+1.6 \mathrm{eV}$. Independent verification of this ion-position independent $q \phi_{\mathrm{SSM}}$ correction is obtained by moving the $\mathrm{Na}^{+}$from the tube interior to an axial position halfway between the tube openings. The energy difference between these configurations is $1.17 \mathrm{eV}$. Thus, $E_{\text {ion }} \sim-1.11 \mathrm{eV}$ indeed reflects the binding energy of $\mathrm{Na}^{+}$in $\mathrm{C}_{48} \mathrm{H}_{24}$. We emphasize that, using non-polarizable force fields,,$\frac{3,5,6}{6}$ the binding energy would be purely dispersive and would at most be a small fraction of an $\mathrm{eV}$.

The binding energy should be independent of the simulation cell size for this isolated SWNT fragment. With a larger, $L=20 \AA$, cell the $q \phi_{\mathrm{SSM}}$ corrected $\mathrm{Na}^{+}\left(\mathrm{Cl}^{-}\right)$ binding energy becomes $1.18 \mathrm{eV}(0.15 \mathrm{eV})$. The small increase compared to $L=15 \AA$ may be due to the polarizability of $\mathrm{C}_{48} \mathrm{H}_{24}$ in the finite simulation cell, which may make $\epsilon_{\infty}$ slightly larger than unity. As $L \rightarrow \infty$, both $q \phi_{\mathrm{SSM}}$ and $\left(\epsilon_{\infty}-1\right)$ vanish as $1 / L^{3}$. Nevertheless, $\left|q \phi_{\mathrm{SSM}}\right|$ remains substantial, $\sim 0.6 \mathrm{eV}$, for the $L=20 \AA$ cell (Table I). It is much larger than that derived for isolated monoatomic ions $\underline{19}$ because of the sheer number of electrons present in $\mathrm{C}_{48} \mathrm{H}_{24}$.

Next, we consider $\mathrm{Na}^{+} / \mathrm{Cl}^{-}$in infinitely long $(6,6)$ and $(18,18)$ SWNT arrays. These models mimic micron-thick carbon nanotube sieves which have been synthesized $\stackrel{8}{8}$ The SWNT's chosen in the present study are metallic and 
exhibit $\epsilon_{\infty} \rightarrow \infty$ in the axial direction. These nanotubes, with cubic unit cell sizes chosen to reflect the SWNT periodicity $\left(14.8 \times 14.8 \times 14.8 \AA^{3}\right.$ and $17.0 \times 17.0 \times 17.0 \AA^{3}$, respectively), are not finite molecules. However, by centering the ion inside the SWNT and the SWNT within the simulation cell, and truncating the electron density at the $z$-direction of the grid boundary - taking care to evenly distribute $\rho(r)$ at the $z$-edges - we have made a physical choice of $\rho(r)$ in the unit cell that exhibits no dipole moment and permits $q \phi_{\mathrm{SSM}}$ corrections. The intrinsic energies of ions as they are displaced from the pore center can then be referenced to the pore center value.

Equation 2 corrects the energies of the isolated ions. Ions inside the SWNT are assumed to have $E_{\text {ewald }}=0$ because of the large $\epsilon_{\infty}$ of the SWNT array. Table \lists the resulting $E_{\text {ion }}$ for $\mathrm{Na}^{+}$and $\mathrm{Cl}^{-}$. $\mathrm{Na}^{+}$and $\mathrm{Cl}^{-}$exhibit large binding energies of 2.9 and $1.8 \mathrm{eV}$ respectively in the narrow $(6,6)$ metallic SWNT arrays. Increasing the Brillouin zone sampling from $\Gamma$-point to a $1 \times 1 \times 10$ Monkhorst-Pack grid leads to changes in $E_{\text {ion }}$ that are less than $0.02 \mathrm{eV}$.

Unlike the $\mathrm{C}_{48} \mathrm{H}_{24}$ fragment, the model SWNT arrays are meant to be periodically replicated in the lateral directions. We consider system size dependences only by increasing the axial dimension of the $(6,6)$ simulation cell to $22.2 \AA$. We find that $E_{\text {ion }}$ are conserved to within $0.03 \mathrm{eV}$ after adding $q \phi_{\mathrm{SSM}}$, which are on the order of several eV (Table I). This suggests that our somewhat arbitary truncation of electronic density at the $z$-edges nevertheless leads to robust $q \phi_{\mathrm{SSM}}$ corrections. Unlike the $\mathrm{Ar}$ and $\mathrm{CH}_{4}$ crystals, where $\Delta \phi=0, E_{\text {ion }}$ cannot yet be referenced to vacuum values because we have not specified the physical interface that will determine $\Delta \phi$. However, the two $q \Delta \phi$ should cancel when we consider the sum of $E_{\text {ion }}$ for the oppositely charge $\mathrm{Na}^{+}$and $\mathrm{Cl}^{-}$. Thus, regardless of the surface terminations, we predict substantial binding energies for ions inside metallic SWNT.

No charge transfer occurs between $\mathrm{Na}^{+}$or $\mathrm{Cl}^{-}$and the $(6,6) \mathrm{SWNT}$ array. DFT/PBE does predict that $\mathrm{Na}^{+}$accepts a small fraction of an electron from the $(18,18)$ array. As a result, we switch to the less electronegative $\mathrm{K}^{+}$, whose lowest unoccupied orbital is found to reside above the $(18,18)$ Fermi level. Thus, $\mathrm{K}^{+}$is representative of a monovalent cation well separated from the SWNT pore surface. It exhibits a still considerable $2.20 \mathrm{eV}$ binding energy inside the wider $(18,18)$ array. $\mathrm{Cl}^{-}$experiences a $1.09 \mathrm{eV}$ binding energy. This slightly overestimates the interaction of this SWNT with a monvalent point anion because DFT/PBE predicts a small charge transfer $(\sim 0.3$ electron $)$ to the SWNT.

Studying ions inside SWNT is pertinent to permeation of electrolytes, where the presence of water strongly stablizes $\mathrm{Na}^{+}$and $\mathrm{Cl}^{+}$and helps prevent electron transfer to or from the nanotubes. How the strongly confined water will screen the induced electrostatic interaction between
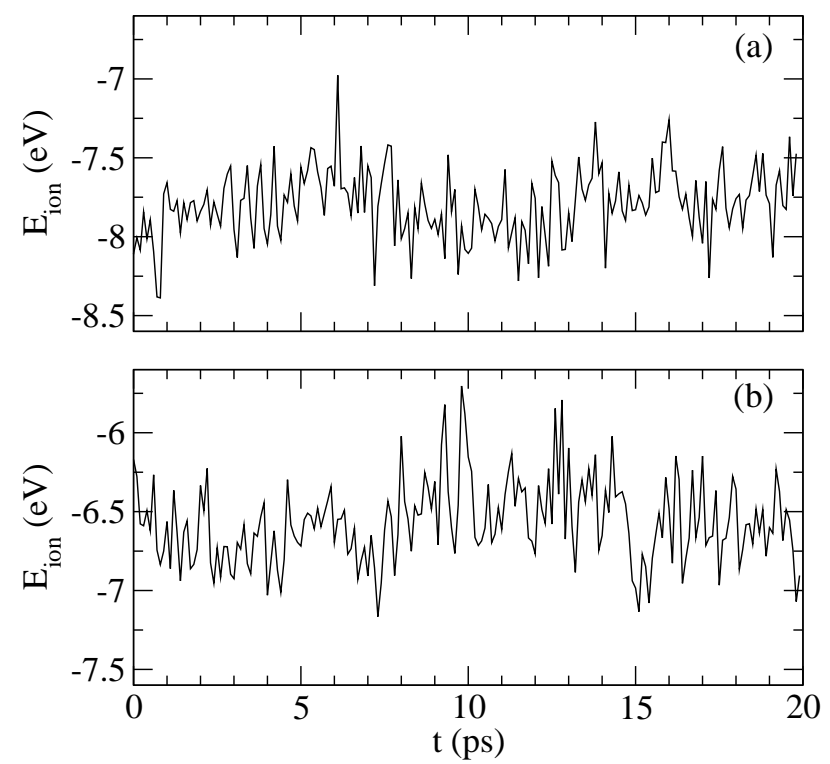

FIG. 2:

$E_{\text {ion }}$ as functions of time for (a) $\mathrm{Na}^{+}$and (b) $\mathrm{Cl}^{-}$in a simulation cell with $32 \mathrm{H}_{2} \mathrm{O}$. There is no significant drift over these short 20 ps trajectories.

ions and SWNT will be examined in the future, but the SWNT polarizability clearly will have a large impact on ion permeation, especially in the narrow $(6,6)$ SWNT that accommodates only a single file water inside,, 5.52

\section{Ions in water}

In this section, we compute the mean $E_{\text {ion }}$ for $\mathrm{Na}^{+}$ and $\mathrm{Cl}^{-}$in liquid water. We apply the maximally localized Wannier function method ${ }^{45,46}$ to decompose electron density on each $\mathrm{H}_{2} \mathrm{O}$. This method correctly locates four Wannier orbital centered within $\sim 1.5 \AA$ of each water oxygen site, corresponding to the two $\mathrm{O}-\mathrm{H}$ covalent bonds and two lone pairs. We choose $\mathrm{O}$ as the molecular center in accordance with classical force field conventions,, 37 and add Ewald corrections (twice that of Eq. 2 as discussed above) and $q \phi_{\mathrm{SSM}}$ to the instantaneous $E_{\text {ion }}$ depicted in Fig. 2. $E_{\text {ion }}$ exhibits little drift over these 20 ps trajectories.

Before we examine $E_{\text {ion }}$ further, the robustness of using Wannier functions to compute $q \phi_{\mathrm{SSM}}$ corrections (Eq. 8) needs to be documented. Over a sample of 14 configurations, evenly distributed over the $\mathrm{Na}^{+}$AIMD trajectory, we find that $q \phi_{\mathrm{SSM}}$ experiences only $\sim 0.01 \mathrm{eV}$ variation at each time step, amounting to a negligable standard deviation of $0.0028 \mathrm{eV}$.

Our maximally localized Wannier functions, by con- 


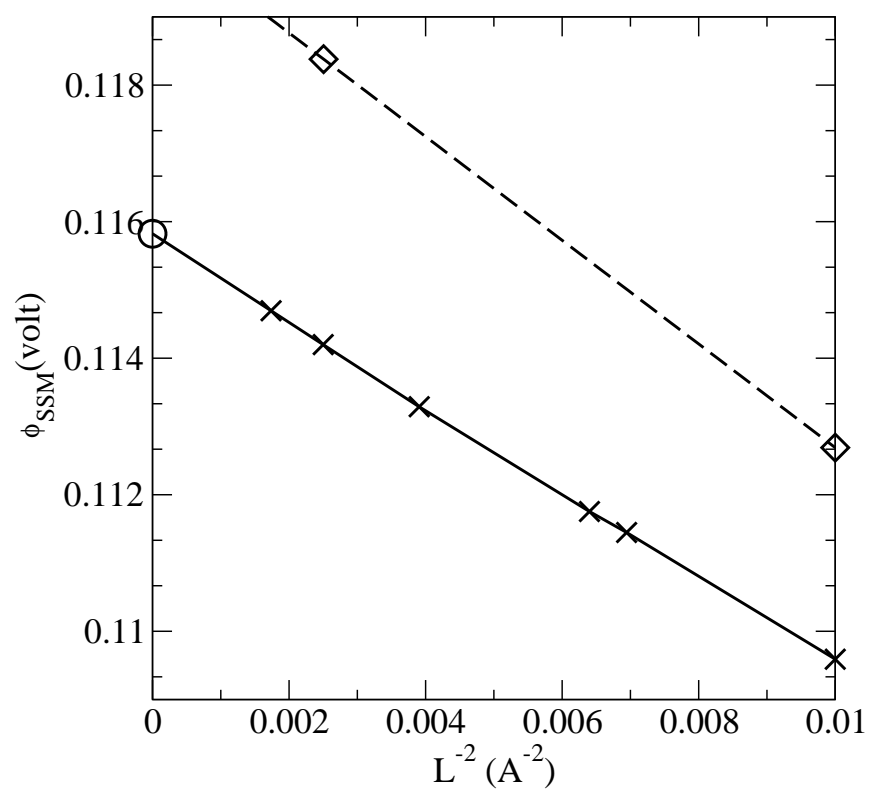

FIG. 3:

$\phi_{\text {SSM }}$ (Eq. 8, in units of volt per $\mathrm{H}_{2} \mathrm{O}$ per $\left.(10 \AA)^{3}\right)$ as the simulation cell size $L$ varies. Crosses: $\phi_{\mathrm{SSM}}$ for an isolated $\mathrm{H}_{2} \mathrm{O}$, using maximally localized Wannier function. Solid line: linear fit to scaling behavior. Circle: $\phi_{\mathrm{SSM}}$ (Eq. 6) computed using $\rho(\mathbf{r})$ directly (without using Wannier functions). Diamond and dashed line: $\phi_{\mathrm{SSM}}$ for $32 \mathrm{H}_{2} \mathrm{O}$

$$
(L=9.9874 \AA) \text { and } 256 \mathrm{H}_{2} \mathrm{O}(L=19.9748 \AA) \stackrel{70}{ }^{70}
$$

struction, depend somewhat on the simulation cell size $\underline{46}$ So do quantities we use to compute $\phi_{\mathrm{SSM}}$ within the Wannier function framework. For a single water molecule in a cubic cell of length $L$, the entire charge density belongs to the one $\mathrm{H}_{2} \mathrm{O}$, and the Wannier function-derived Eq. 6 can be compared with the value obtained from numerical integration of $\rho(r)$. Figure 3 shows that the Wannier-derived $\phi_{\text {SSM }}$ convergence $\propto L^{-2} ; \phi_{\text {SSM }}$ exhibits an empirical correction $+0.623 / L^{2}$ volt- $\AA^{2}$ per $\mathrm{H}_{2} \mathrm{O}$ in a $(10 \AA)^{3}$ unit cell, or $+623 / L^{5}$ volt- $\AA^{5}$ per $\mathrm{H}_{2} \mathrm{O}$. The scaling is consistent with analysis of $\langle\mathbf{r}\rangle$ and $\left\langle r^{2}\right\rangle$ described in the Appendix. (Recall $\phi_{\mathrm{SSM}}$ scales as $1 / \Omega$ for isolated molecules.) Numerical integration over $\rho(r)$ yields $L$-independent $\phi_{\mathrm{SSM}}$. Its value agrees to within $0.01 \%$ of the Wannier $\phi_{\mathrm{SSM}}$ when the latter is extrapolated to $L \rightarrow \infty$. This extrapolation increases $q \phi_{\mathrm{SSM}}$ computed in a $L \sim 10 \AA$ cubic cell by $\sim 5.5 \%$. For water at $1.0 \mathrm{~g} / \mathrm{cc}$ density $\left(32 \mathrm{H}_{2} \mathrm{O}\right.$ molecules for this cell size), this increase amounts to 0.22 volt.

Next, we show that the presence of multiple molecules does not substantially change the $L$-dependence. We expand a $L=9.9874 \AA$ box of 32 water molecules into a $2 \times 2 \times 2$ supercell, and compare the difference in $\phi_{\mathrm{SSM}}$ for this water configuration. The $L=19.9758 \AA$ supercell

\begin{tabular}{||c|c|c||}
\hline ion & $N_{\mathrm{w}}$ & $\left\langle E_{\text {ion }}\right\rangle$ \\
\hline $\mathrm{Cl}^{-}$ & 32 & $-6.57(-2.60)$ \\
$\mathrm{Cl}^{-}$ & 64 & $-6.27(-2.29)$ \\
$\mathrm{Cl}^{-\dagger}$ & 256 & $-7.39(-8.23)$ \\
$\mathrm{Na}^{+}$ & 32 & $-7.85(-11.54)$ \\
$\mathrm{Na}^{+}$ & 64 & $-7.70(-11.36)$ \\
$\mathrm{Na}^{+\dagger}$ & 256 & $-10.01(-9.17)$ \\
\hline
\end{tabular}

TABLE II: Mean $E_{\text {ion }}$ in eV, accumulated using the PW91 functional with (without) the $q \phi_{\mathrm{SSM}}$ correction. $N_{w}$ is the number of water molecules in the simulation cell. ${ }^{\dagger} \mathrm{SPC} / \mathrm{E}$ classical force field results (Table 4, Ref. 23), which are independent of simulation cell size.

$\phi_{\mathrm{SSM}}$ exceeds the $L=9.9874 \AA$ value by $5 \%$. According to the scaling curve in Fig. 3 , the Wannier function approach yields a $4.2 \%$ difference between $L=20$ and $L=10 \AA$ for an isolated $\mathrm{H}_{2} \mathrm{O}$. This suggests that extrapolating $q \phi_{\mathrm{SSM}}$ computed for a $L \sim 10 \AA^{3}$ box of liquid water to $L \rightarrow \infty$ at $1.0 \mathrm{~g} / \mathrm{cc}$ density, using the $L$-scaling for a single $\mathrm{H}_{2} \mathrm{O}$, should only lead to a small systematic error of about $(5-4.2) / 5 \times 0.22$ volt, or about 0.035 volt.

Having shown that $q \phi_{\mathrm{SSM}}$ corrections generated using maximally Wannier functions are robust, and that the errors are well-controlled, we return to $E_{\text {ion }}$ in liquid water (Fig. 2). $E_{\text {ion }}$ values averaged over at least $20 \mathrm{ps}$ are listed in Table III $q \phi_{\mathrm{SSM}}$ is computed using Eq. 8 in the presence of the ion, with $q \phi_{\mathrm{SSM}}$ for the isolated $\mathrm{Cl}^{-}$accounted for. Removing the ion before computing $q \phi_{\mathrm{SSM}}$ changes this term by less than $0.01 \mathrm{eV}$. Overall, $q \phi_{\mathrm{SSM}}$ is large - on the order of several $\mathrm{eV}$ - for the water-filled simulation cell. $\mathrm{Na}^{+}$and $\mathrm{Cl}^{-}$exhibit a $1.28 \mathrm{eV}$ asymmetry in $E_{\text {ion }}$. To properly compare this with force field results, we also apply the $q \phi_{\mathrm{SSM}}$ correction to SPC/E water model used in Ref ${ }^{23}$ with oxygen as the molecular center. Because of the SPC/E charge distribution, this increases (decreases) the magnitude of $E_{\text {ion }}$ for $\mathrm{Na}^{+}$ $\left(\mathrm{Cl}^{-}\right)$. These force field based $\mathrm{Na}^{+}$and $\mathrm{Cl}^{-} E_{\text {ion }}$ now exhibit a $2.39 \mathrm{eV}$ difference, which is substantially larger than the DFT/PW91 result. The sum of the PW91 $\mathrm{Cl}^{-}$ and $\mathrm{Na}^{+} E_{\text {ion }},-14.42 \mathrm{eV}$, is also substantially smaller than the $-17.40 \mathrm{eV}$ predicted using the non-polarizable SPC/E water model $\stackrel{23}{=}$ The comparisons depend on the force field used.

$E_{\text {ion }}$ (Eq. 1) is the end point of thermodynamic integration (TI) of ion hydration in systems without electronic polarizability. With polarizable water force fields, it should be replaced by $\left\langle d E_{\text {ion }} / d\left(\lambda q_{\text {ion }}\right)\right\rangle_{\lambda=1}$, where $q_{\text {ion }}$ is the charge of a non-polarizable ion force field. AIMD treats water (and ion) polarizability, but this formulation is in general not applicable because $q_{\text {ion }}$ is set by total number electrons in the system, and the highest occupied and lowest unoccupied orbitals may not generally reside on the ion. Instead, a quantum mechanics/molecular mechanics (QM/MM) approach with the 
ion treated classically, followed by free energy perturbation to replace the MM ion by a DFT ion, may be a preferred thermodynamic route to compute AIMD ion hydration free energies.

Thus, while the ion hydration free energies reported in Ref. 23 are in reasonable agreement with experiments and the DFT results are smaller and might suggest that DFT/PW91 underestimates monovalent ion energetics in water, comparing the end-point integrand value of a thermodynamic integration formula between non-polarizable force fields and fully polarizable, DFT-based AIMD methods may be misleading. Furthermore, thermodynamic theories that incorporate DFT ion-water cluster energies and dielectric continuum approaches have yielded good agreement with experiments ${ }^{71}$

Classical force field intrinsic $E_{\text {ion }}$ exhibit $O(1) \mathrm{kcal} / \mathrm{mol}$ cell size dependences after correcting for image and background charge contributions. ${ }^{23}$ However, there have been recent suggestions that AIMD simulations exhibit larger cell size dependences for charged species $\underline{16}$ Thus, we also consider a $\mathrm{Na}^{+} / \mathrm{Cl}^{-}$plus $64 \mathrm{H}_{2} \mathrm{O}$ AIMD simulation at $1.0 \mathrm{~g} / \mathrm{cc} \mathrm{H}_{2} \mathrm{O}$ density for $20 \mathrm{ps}$. We find that $E_{\text {ion }}$ changes by $+0.15 \mathrm{eV}(+0.30 \mathrm{eV})$ for $\mathrm{Na}^{+}\left(\mathrm{Cl}^{-}\right)$. This amounts to a $3.5(6.9) \mathrm{kcal} / \mathrm{mol} L$-dependence.

Are these $L$-dependences statistically significant? It is difficult to estimate the uncertainty in $E_{\text {ion }}$ using our short, $\sim 20$ ps AIMD trajectories (Fig. 22). Using the fact a 1 ns classical force field molecular dynamics simulation typically yields a standard deviation of $\sim 0.5 \mathrm{kcal} / \mathrm{mol}$ in $E_{\text {ion }}$, we estimate that our 20 ps AIMD simulations have $3 \mathrm{kcal} / \mathrm{mol}$ statistical uncertainties. In this sense, the $E_{\text {ion }}$ difference between the $32-$ and $64-\mathrm{H}_{2} \mathrm{O}$ cells is within the expected statistical errors. This test seems to suggest that our use of twice the value of Eq. $2.2 \times$ $2.04 \mathrm{eV}$, to correct the monopole-image interaction leads to a reasonable system size convergence (see Sec. IIB). However, the AIMD solvent electronic polarizability also plays a role in modifying the system size dependence seen in Ref. 23. Further studies on ion hydration free energies are needed to address this issue.

\section{CONCLUSIONS}

In this paper, we apply DFT methods to compute the energies of ions in condensed phase systems. In general, due to the ambiguity in the average intrinsic electrostatic potential $\phi_{o}$ arising from periodic boundary conditions and Fourier transforms/Ewald sums, this energy is ill-defined. It even depends on the number of electrons (valence vs. core) and computational protocol. For two types of systems which are pertinent to ion permeation in water-filled nanopores, we demonstrate that the intrinsic ion energy can nevertheless be reported. The key is to apply the "spherical second moment" correction $\left(\phi_{\mathrm{SSM}}\right) \stackrel{13,37}{, 3}$ in addition to the well-known Ewald correction pertinent to a charged simulation cell with a uniform neutralizing background 19,23 A physical choice of unit cell boundary is involved in this correction.

The first type of systems consists of molecules or nanotubes whose unit cell exhibits no permanent dipole moment. This enables relatively unambiguous estimates of $q \phi_{\mathrm{SSM}}$, and yields ion energies that can be referenced to the vacuum phase. Note that the ion energy will still mathematically depend on the shape of the crystal if the unit cell contains a non-vanishing quadrupole moment. Surface potential terms may also contribute if the material surface is chemically different from the bulk region. Fortunately, interfacial effects are additive to intrinsic ion energies. With this approach, and choosing cell boundaries that do not split up nanopore electronic densities in radial directions, we have computed the interaction between an ion and model molecular crystals and carbon nanotubes. The intrinsic binding energies for metallic $(6,6)$ tubes are found to be 2.9 and $1.8 \mathrm{eV}$ for $\mathrm{Na}^{+}$and $\mathrm{Cl}^{-}$, respectively. Even the much wider $(18,18)$ SWNT's exhibit binding energies of 2.2 and (a slightly overestimated) $1.1 \mathrm{eV}$ for monovalent cations and anions, respectively. This suggests that the proposed use of vertically aligned SWNT sieves as ion rejection membranes $3, \frac{3,6}{6}$ may need to be re-examined.

The second type of systems consists of liquid-state simulation cells with no obvious symmetry or other means to eliminate the net unit cell dipole moment. The philosophy is different here, and follow formulations derived for classical water force fields, but $q \phi_{\mathrm{SSM}}$ corrections are still useful because of orientational self-averaging in the liquid state. Here we apply maximally localized Wannier functions to decompose charge densities onto molecules. $\phi_{\mathrm{SSM}}$ will depend on the choice of molecular center, which needs to be consistently defined to compare with classical force field results. It also involves the physical choice that each water molecule is preserved intact with no splitting of electron density across cell boundaries. With consistent $\phi_{\mathrm{SSM}}$ protocols and using a 32 -water cell, we find that $\mathrm{Na}^{+}$and $\mathrm{Cl}^{-}$exhibit ion energies (i.e., hydration energies neglecting solvent reorganization) of -7.9 and $6.6 \mathrm{eV}$ respectively. These are considerably smaller than classical force field ion energies of -9.0 and $-7.1 \mathrm{eV} \underline{\underline{23}}$ Using a large simulation cell containing $64 \mathrm{H}_{2} \mathrm{O}$ molecules leads to 0.15 and $0.30 \mathrm{eV}$ reductions in the ion energies. These differences are within the statistical uncertainties of the simulations. Note that our "ion energy" in the aqueous phase neglects solvent reorganization and is not a measurable quantity. It also differs from the end-point of the thermodynamic integration formula for the hydration free energy because of electronic polarizability issues. Nevertheless, with consistent $\phi_{\mathrm{SSM}}$ corrections, $\mathrm{Na}^{+}$and $\mathrm{Cl}^{-}$ion energies become comparable, do not contain ambiguities associated core electrons in pseudopotentials, and their asymmetry is now comparable to force field predictions. The ability to correct for $\phi_{o}$ is important 
for future ion hydration free energy calculations using AIMD in conjunction with thermodynamic integration methods.

Looking ahead, it is tantalizing to contemplate how the methods described in this work can be applied to compute ion energies in crystalline systems and nanoporous solids. ${ }^{4}$

\section{Acknowledgement}

We thank Peter Schultz, Normand Modine, and Susan Rempe for useful discussions and suggestions. Sandia is a multiprogram laboratory operated by Sandia Corporation, a Lockheed Martin Company, for the U.S. Department of Energy's National Nuclear Security Administration under contract DE-AC04-94AL8500. One of us (MM) was supported by the Austrian Fonds zur Förderung der wissenschaftlichen Forschung within the START grant.

\section{APPENDIX: MAXIMALLY LOCALIZED WANNIER FUNCTIONS.}

The maximally localized Wannier functions $W_{n}$ are defined as linear combinations of the $N_{\text {occ }}$ selfconsistent Bloch wave functions $\Psi_{n}$ that make up the space of occupied orbitals,

$$
\left|W_{n}\right\rangle=\sum_{n^{\prime}=1}^{N_{\text {occ }}} U_{n n^{\prime}}\left|\Psi_{n^{\prime}}\right\rangle,
$$

where $U$ is the $N_{\text {occ }} \times N_{\text {occ }}$ unitary transformation matrix that minimizes the spread functional,

$$
\Xi[U]=\sum_{n=1}^{N_{\text {occ }}}\left(\left\langle W_{n}\left|r^{2}\right| W_{n}\right\rangle-\left\langle W_{n}|\mathbf{r}| W_{n}\right\rangle^{2}\right),
$$

which provides a measure for the degree of spatial localization of the Wannier functions. (Since the implementation of the maximally localized Wannier functions in VASP is limited to a $\Gamma$-point only sampling of the Brillouin zone, we have dropped the usual k-point index.)

Following Berghold et al $\underline{\underline{46}}$ we define

$$
z_{I, n}=\left\langle W_{n}\left|e^{i \mathbf{G}_{I} \cdot \mathbf{r}}\right| W_{n}\right\rangle,
$$

and rewrite Eq. (A.2) as

$$
\Xi[U]=\frac{1}{(2 \pi)^{2}} \sum_{n=1}^{N_{\text {occ }}} \sum_{I=1}^{6} \omega_{I}\left(1-\left|z_{I, n}\right|^{2}\right)+O\left(\mathbf{G}_{I}^{2}\right),
$$

where $\mathbf{G}_{1}=(1,0,0), \mathbf{G}_{2}=(0,1,0), \mathbf{G}_{3}=(0,0,1), \mathbf{G}_{4}=$ $(1,1,0), \mathbf{G}_{5}=(1,0,1)$, and $\mathbf{G}_{6}=(0,1,1)$, with respect to the basis of reciprocal lattice vectors. The weights $\omega_{I}$ are defined as in Appendix A of Ref. 46 .

In the PAW method ${ }^{49,50}$ the one-electron wave functions $\Psi_{n}$ are derived from the pseudo-wave-functions $\widetilde{\Psi}_{n}$ by means of a linear transformation

$$
\left|\Psi_{n}\right\rangle=\left|\widetilde{\Psi}_{n}\right\rangle+\sum_{k}\left(\left|\phi_{k}\right\rangle-\left|\widetilde{\phi}_{k}\right\rangle\right)\left\langle\widetilde{p}_{k} \mid \widetilde{\Psi}_{n}\right\rangle,
$$

The pseudo-wave-functions $\widetilde{\Psi}_{n}$ are the variational quantities of the PAW method, and are expanded in reciprocal space using plane waves. The index $k$ is a shorthand for the atomic site $\mathbf{R}_{k}$, the angular momentum quantum numbers $l_{k}$ and $m_{k}$, and the reference energy $\epsilon_{k}$. The all-electron partial waves $\phi_{k}$ are solutions of the radial Schrödinger equation for a non-spin-polarized reference atom at specific energies $\epsilon_{k}$ and for a specific angular momentum $l_{k}$. The pseudo-partial-waves $\widetilde{\phi}_{k}$ are equivalent to the all-electron partial waves outside a core radius $r_{c}$ and match continuously onto $\phi_{k}$ inside the core radius. The projector functions $\widetilde{p}_{k}$ are dual to the pseudo-partialwaves (i.e., $\left.\left\langle\widetilde{p}_{k} \mid \widetilde{\phi}_{l}\right\rangle=\delta_{k l}\right)$.

Following Ferretti et al.,$\underset{72}{n}$ we use Eqs. (A.1) and (A.5), to rewrite Eq. A.3 as

$$
\begin{aligned}
z_{I, n} & =\left\langle\widetilde{W}_{n}\left|e^{i \mathbf{G}_{I} \cdot \mathbf{r}}\right| \widetilde{W}_{n}\right\rangle+\sum_{k l}\left\langle\widetilde{W}_{n} \mid \widetilde{p}_{k}\right\rangle\left\langle\widetilde{p}_{l} \mid \widetilde{W}_{n}\right\rangle \\
& \times \int e^{i \mathbf{G}_{I} \cdot \mathbf{r}}\left[\phi_{k}(\mathbf{r}) \phi_{l}(\mathbf{r})-\widetilde{\phi}_{k}(\mathbf{r}) \widetilde{\phi}_{l}(\mathbf{r})\right] d \mathbf{r} .
\end{aligned}
$$

In our implementation, the integral over the partial waves in Eq. A.6) is approximated by

$$
\sum_{L} \int_{\Omega_{\mathbf{R}_{k}}} \hat{Q}_{k l}^{L}(\mathbf{r}) e^{i \mathbf{G}_{I} \cdot \mathbf{r}} d \mathbf{r}
$$

where $\hat{Q}_{k l}^{L}(\mathbf{r})$ is given by Eq. (27) of Ref. 50 , and $\Omega_{\mathbf{R}_{k}}$ is the PAW sphere at atomic site $\mathbf{R}_{k}$. [Note that the double sum in Eq. A.6 is limited to be site-diagonal, i.e., $\mathbf{R}_{k}=\mathbf{R}_{l}$.] An exact expression for the integral in Eq. (A.6) can be found in App. B of Ref. 72.

Thus defined within the PAW formalism, the spread functional $\Xi[U]$ is minimized with respect to $U$ by means of the two-by-two orbital rotation method introduced by Edminston and Ruedenberg (see Sec. III A. of Ref. 46 and references therein). Once the maximally localized Wannier functions are constructed, we use

$$
\left\langle r^{2}\right\rangle_{n}=\frac{1}{(2 \pi)^{2}} \sum_{I=1}^{6} \omega_{I}\left(1-\left|z_{I, n}\right|^{2}\right)+\langle\mathbf{r}\rangle_{n}^{2}+O\left(\mathbf{G}_{I}^{2}\right)
$$

and

$$
\langle\mathbf{r}\rangle_{n}=\left\langle W_{n}|\mathbf{r}| W_{n}\right\rangle=\frac{1}{2 \pi} \sum_{I=1}^{3} \mathbf{a}_{I} \Im \ln z_{I, n}+O\left(\mathbf{G}_{I}^{2}\right),
$$


where $\mathbf{a}_{I}$ are the real space lattice vectors, to replace the electronic part of the integrals $\int \rho_{m}(\mathbf{r}) \mathbf{r} d \mathbf{r}$ and $\int \rho_{m}(\mathbf{r}) r^{2} d \mathbf{r}$, in Eqs. (77) and (8), by the sums $\sum_{i} e\langle\mathbf{r}\rangle_{i}$ and $\sum_{i} e\left\langle r^{2}\right\rangle_{i}$ taken over the set of Wannier functions associated with molecular center $m$.
Note that Eqs. (A.8) and (A.9) are exact to order $O\left(\mathbf{G}_{I}^{2}\right)$, which for simple cubic supercells of lattice parameter $L$, means the expressions for $\left\langle r^{2}\right\rangle_{n}$ and $\langle\mathbf{r}\rangle_{n}$ converge as $L^{-2}$ in the limit of large $L$ (see Fig. 3).
1 L. X. Benedict, S. G. Louie, and M. L. Cohen, Phys. Rev. B 52, 8541 (1995).

2 B. Kozinsky and N. Marzari, Phys. Rev. Lett. 96, 166801 (2006).

3 K. Leung, S. B. Rempe, and C. D. Lorenz, Phys. Rev. Lett. 96, 095504 (2006).

4 M. Nyman, A. Tripathi J. B. Parise, R. S. Maxwell, and T. M. Nenoff, J. Am. Chem. Soc. 124, 1704 (2002).

5 A. Kalra, S. Garde, and G. Hummer, Proc. Natl. Acad. Sci. 100, 10175 (2003).

${ }^{6}$ C. Peter and G. Hummer, Biophys. J. 89, 2222 (2005).

7 J. K. Holt, H. G. Park, Y. M. Wang, M. Stadermann, A. B. Artyukhin, C. P. Grigoropoulos, A. Noy, and O. Bakajin, Science 312, 1034 (2006).

8 B. J. Hinds, N. Chopra, T. Rantell, R. Andrews, V. Gavalas, and L. G. Bachas, Science 303, 62 (2004).

9 Y.-B. Jiang, N. Liu, H. Gerung, J. L. Cecchi, and C. J. Brinker, J. Am. Chem. Soc. 128, 11018 (2006).

10 L. Kleinman, Phys. Rev. B 24, 7412 (1981).

11 S. W. de Leeuw, J. W. Perram, and E. R. Smith, Proc. Roy. Soc. A 373, 27 (1980).

12 A. Redlack and J. Grindlay, J. Phys. Chem. Solids 36, 73 (1975).

13 V. R. Saunders, C. Freyria-Fava, R. Dovesi, L. Salasco, and C. Roetti, Mol. Phys. 77, 629 (1992).

14 P. Hunt and M. Sprik, Chem. Phys. Chem. 6, 1805 (2005).

15 J. Blumberger and M. Sprik, J. Phys. Chem. B 109, 6793 (2005).

16 J. Blumberger, L. Bernasconi, I. Tavernelli, R. Vuilleumier, M. Sprik, J. Am. Chem. Soc. 1263928 (2004).

17 N. D. Lang and W. Kohn, Phys. Rev. B 3, 1215 (1971).

18 A. Y. Lozovoi, A. Alavi, J. Kohanoff, and R. M. LyndenBell, J. Chem. Phys. 115, 1661 (2001).

19 G. Makov and M. C. Payne, Phys. Rev. B 51, 4014, (1995).

20 P. A. Schultz, Phys. Rev. Lett. 84, 1942 (2000).

${ }^{21}$ R. N. Euwema and G. T. Surratt, J. Phys. Chem. Solids 36, 67 (1975).

22 In this work, we will focus on Ewald sum-based classical force field calculations.

${ }^{23}$ G. Hummer, L. R. Pratt, and A. E. Garcia, J. Phys. Chem. 100, 1206 (1996).

24 G. Hummer, L. R. Pratt, and A. E. Garcia, J. Chem. Phys. 107, 9275 (1997).

25 G. Hummer, L. R. Pratt, A. E. Garcia, B. J. Berne, and S. W. Rick, J. Phys. Chem. B 101, 3017 (1997).

${ }^{26}$ G. Hummer, L. R. Pratt, and A. E. Garcia, J. Phys. Chem. A 102, 7885 (1998).

27 H. S. Ashbaugh and R. H. Wood, J. Chem. Phys. 106, 8135 (1997).

28 T. Darden, D. Pearlman, and L. G. Pedersen, J. Chem. Phys. 109, 10921 (1998).

${ }^{29}$ R. M. Lynden-Bell and J. C. Rasaiah, J. Chem. Phys. 107,
1981 (1997).

${ }^{30}$ F. Figueirido, G. S. Del Buono, and R. M. Levy, J. Phys. Chem. B 101, 5622 (1997).

31 P. H. Hünenberger and J. A. McCammon, J. Chem. Phys. 110, 1856 (1999).

32 A. Grossfield, P.-Y. Ren, and J. W. Ponder, J. Am. Chem. Soc. 125, 15671 (2003).

33 M. P. Allen and D. J. Tildesley, Computer Simulation of Liquids (Oxford University Press, New York, 1987).

34 Y. Marcus, Biophys. Chem. 51, 111 (1994), and references therein.

35 M. D. Tissandier, K. A. Cowen, W. Y. Feng, E. Grunlach, M. H. Cohen, A. D. Earhart, J. V. Coe, and T. R. Tuttle, J. Phys Chem. A 102, 7787 (1998).

${ }^{36}$ L. R. Pratt, J. Phys. Chem. 96, 25 (1992), and references therein.

37 M. A. Wilson, A. Pohorille, and L. R. Pratt, J. Chem. Phys. 90, 5211 (1989).

38 M. A. Wilson, A. Pohorille, and L. R. Pratt, J. Phys. Chem. 91, 4873 (1987).

39 V. P. Sokhan and D. J. Tildesley, Mol. Phys. 92, 625 (1997)

40 L. X. Dang and T.-M. Chang, J. Phys. Chem. B 106, 235 (2002).

41 For the recently devised TIP5P force field [M. W. Mahoney and W. L. Jorgensen, J. Chem. Phys. 112, 8910 (2000)], however, this contribution is quite different from the more widely used SPC/E or TIP3P/TIP4P series.

42 P. C. Rusu and G. Brocks, Phys. Rev. B 74, 073414 (2006).

43 Y. Zhou, G. Stell, and H. L. Friedman, J. Chem. Phys. 89, 3836 (1988).

44 E. A. Guggenheim, Thermodynamics (North-Holland, Amsterdam, 1967) Ch. 8.

45 N. Marzari and D. Vanderbilt, Phys. Rev. B 56, 12847 (1997).

46 G. Berghold, C. J. Mundy, A. H. Romero, J. Hutter, and M. Parrinello, Phys. Rev. B 61, 10040 (2000).

47 J. Åqvist and T. Hansson, J. Phys. Chem. B 102, 3837 (1998).

${ }^{48}$ G. Hummer, L. R. Pratt, A. E. Garcia, S. Garde, B. J. Berne, and S. W. Rick, J. Phys. Chem. B 102, 3841 (1998).

49 P. E. Blöchl, Phys. Rev. B, 50, 17953 (1994).

50 The VASP implementation is discussed in G. Kresse and D. Joubert, Phys. Rev. B 59, 1758 (1999).

51 G. J. Martyna and M. E. Tuckerman, J. Chem. Phys. 110, 2810 (1999).

52 G. Hummer, J. C. Rasaiah, and J. P. Noworyta, Nature 414, 188 (2001).

53 J. D. Jackson, Classical Electrodynamics (Wiley, New York, 1975) Ch. 4.

${ }^{54}$ F. E. Harris, Theoretical Chemistry: Advances and Per- 
spectives, ed. H. Eyring and D. Henderson, (Academic Press) 1, 147 (1975).

55 There have been recent controversies regarding whether the intrinsic ion hydration free energies should be reported with or without the $q \phi_{\mathrm{SSM}}$ correction $.56,57$ This seems to amount to shifting $q \phi_{\mathrm{SSM}}$ from $q \Delta \phi$ (Eq. 9) to Eq. 1. The free energy change of taking an ion from vacuum, through the interface, into liquid, remains necessarily the same. However, this shift appears necessary for AIMD to ensure that reported AIMD ion energies are independent of whether core electrons are used. Note that, when using Ewald sums, "molecular" or "atomic" based definitions of bulk water charge distributions ${ }^{56}$ yield identical electrostatic potentials. ${ }^{25,27}$

56 M. A. Kastenholz and P. H. Hünenberger, J. Chem. Phys. 124, 124106 (2006).

57 M. A. Kastenholz and P. H. Hünenberger, J. Chem. Phys. 124, 224501 (2006).

${ }^{58}$ I. F. W. Kuo and C. J. Mundy, Science 303, 658 (2004).

59 M. J. McGrath, J. I. Siepmann, I. F. W. Kuo, C. J. Mundy, I. VandeVondele, J. Hutter, F. Mohamed, and M. Krack, J. Phys. Chem. A 110, 640 (2006).

60 G. Kresse and J. Furthmüller, Phys. Rev. B 54, 11169 (1996), Comput. Mater. Sci. 6, 15 (1996).

61 D. Vanderbilt, Phys. Rev. B 41, 7892 (1990).

62 J. P. Perdew and Y. Wang, Phys. Rev. B 45, 13244 (1992).

63 J. P. Perdew, K. Burke, K. M. Ernzerhof, Phys. Rev. Lett.
77, 3865 (1996).

64 D. Asthagiri, L. R. Pratt, J. D. Kress, Phys. Rev. E 68 041505 (2003).

65 J. C. Grossman, E. Schwegler, E. W. Draeger, F. Gygi, G. Galli, J. Chem. Phys. 120, 300 (2004).

66 J. VandeVondele, F. Mohamed, M. Krack, J. Hutter, M. Sprik, M. Parrinello, J. Chem. Phys. 122014515 (2005)

67 In reality, $\epsilon_{\infty}$ should be slightly larger than unity because of the polarizable molecules in the cell, even though they have a large HOMO-LUMO gap and their density is low. If the DFT $\epsilon_{\infty}=1.05$, then according to Eq. $2 E_{\text {ion }}$ for both ions would be underestimated by $0.06 \mathrm{eV}$. The $\epsilon_{\infty}=1$ assumption should not alter the difference between these $E_{\text {ion }}$.

68 M. Sprik, M. L. Klein, and K. Watanabe, J. Phys. Chem. 94, 6483 (1990).

69 P. Paricaud, M. Predota, A. A. Chialvo, and P. T. Cummings, J. Chem. Phys. 122, 244511 (2005).

70 At each cell size, the Eq. 8 correction is larger for in the condensed than the gas phase because hydrogen bonding alters the charge distributions.

71 S. B. Rempe, D. Asthagiri, and L. R. Pratt, Phys. Chem. Chem. Phys. 6, 1966 (2004).

72 A. Ferretti, A. Calzolari, B. Bonferroni, and R. Di Felice, J. Phys. Condens. Matter 19, 036215 (2007). 